\title{
Phonon-drag thermopower of lateral superlattices: the role of anisotropic scattering
}

\author{
D Uzur ${ }^{1}$, A Nogaret ${ }^{1}$, A G Pogosov ${ }^{2}$, H E Beere ${ }^{3}$ and D A Ritchie ${ }^{3}$ \\ ${ }^{1}$ Department of Physics, University of Bath, Claverton Down, Bath BA2 7AY, UK \\ 2 Institute of Semiconductor Physics, Siberian Branch of the Russian Academy of Science, \\ 630090 Novosibirsk, Russia \\ ${ }^{3}$ Cavendish Laboratory, University of Cambridge, Cambridge CB3 OHE, UK \\ E-mail: A.R.Nogaret@bath.ac.uk
}

Received 10 July 2003, in final form 12 September 2003

Published 3 October 2003

Online at stacks.iop.org/JPhysCM/15/6985

\begin{abstract}
We have measured the phonon-drag thermopower of a periodically modulated two-dimensional electron gas, and report on a complex series of oscillations developing in the presence of a perpendicular magnetic field. At low electron density these oscillations are in phase with the commensurability resistance oscillations, however they become increasingly antisymmetrical with respect to $B$ at higher electron density. We are able to explain this magnetic field dependence by proposing that the periodic potential lowers the symmetry of electron-phonon interaction. We calculate the thermopower by solving the Boltzmann equation given an electron-phonon scattering term with two-fold symmetry. Our fit of the experimental curves shows that the Brillouin zone folding enhances the electron-phonon scattering rate by a factor of two along the periodic potential and that a small misalignment $\left(6^{\circ}\right)$ of the heat gradient with the direction of the periodic potential is sufficient to explain the antisymmetrical oscillations. Our experiment demonstrates phonon drag as a very sensitive tool to probe the electronic and vibronic anisotropy in mesoscopic systems.
\end{abstract}

\section{Introduction}

The resistance [1] and the diffusion thermopower [2-6] of mesoscopic systems has been investigated intensively, yet no experiment to date has revealed the benefits of using the phonon-drag thermopower (PDTP) to study confined electron and phonon systems. This lack of interest can be partly traced to earlier studies of bulk semiconductors [7] and twodimensional electron gases (2DEGs) [8] that showed the phonon drag to depend only on the electron-phonon coupling and phonon parameters, not the electrical conductivity (or its derivative) as in the resistance (or the diffusion thermopower, respectively). It is, for example, well known that magnetic field dependent phenomena such as weak localization and universal 
conductance fluctuations cannot be measured by phonon-drag experiments [9]. Of course, at higher magnetic fields, Landau levels produce oscillations in the electron-phonon scattering rate as they cross the Fermi level. This allowed PDTP measurements of the integer and the fractional quantum Hall effect [10]. However, in the regime of low magnetic fields that is relevant to us here, the Fermi density of states is constant and therefore quenches all magnetic field dependence. A periodic electrostatic modulation only adds corrective terms in the conductivity tensor [11-13] and since the PDTP is independent of the conductivity, no magnetic field dependence is, a priori, expected in such systems either. This is what this paper is set to investigate.

The principle of the experiment is simple. A temperature gradient is applied to the lattice and phonons that propagate away from the heat source transfer part of their momentum to conduction electrons. The resulting thermoelectric current carries electrons towards the cold source. These charges cannot leave the sample since no net current is applied $(J=0)$, hence the cold source charges negatively with respect to the hot source and an electric field is established that prevents further charge build-up. This electric field is proportional to the temperature gradient with $E=S^{\circ} \nabla T$ where $S^{\circ}$ is the thermopower that we measure. The PDTP of bulk systems was shown to be: $S^{\circ}=-\frac{\Lambda v}{\mu_{\mathrm{ep}} T}$ [9] where $\Lambda$ is the phonon mean free path, $v$ the sound velocity in acoustic branches and $\mu_{\mathrm{ep}}$ the mobility limited by the electron-phonon scattering time $\tau_{\mathrm{ep}}$.

We report on the PDTP of a periodically modulated 2DEG measured as a function of a perpendicular magnetic field, $B$. Commensurability oscillations are obtained which are in phase with the well known resistance oscillations. These oscillations become increasingly antisymmetrical with respect to the change of sign of $B$ as the electron density increases. We trace these oscillations to anisotropy in the electron-phonon interaction. The 1D potential modulation fragments the Fermi surface into Brillouin minizones introducing a two-fold anisotropy in the electron-phonon interaction. We find that Brillouin zone folding enhances acoustic phonon scattering along the superlattice because its matrix element diverges for transitions between the edges of the Fermi surface (Kohn anomalies). The anisotropy breaks the co-linearity between the temperature gradient and the electric field which explains the magnetic field dependence of the PDTP.

The paper is organized as follows. The second section describes the sample preparation and the experimental method. The magneto-thermopower curves are analysed and compared to the commensurability oscillations in the resistance. The third section presents the theoretical model of the PDTP and discusses the calculated magnetic field dependence. We used this theory to fit the experimental thermopower and extract the magnitude and direction of the electron-phonon anisotropy in the superlattice. The results and their meaning are discussed together with the success and the limitations of the present theoretical framework. The fourth section summarizes our results.

\section{Experimental details}

Hall bar devices were defined by optical lithography in a $\left(\mathrm{GaAs} / \mathrm{Al}_{0.3} \mathrm{Ga}_{0.7} \mathrm{As}\right)$ modulation doped heterostructure. The $1 \mathrm{~mm}$ spacing between the voltage probes shown in figure 1(a) was chosen to be much larger than the energy relaxation length of hot electrons generated by the heating line. An array of dysprosium stripes was fabricated by electron beam lithography in order to modulate the 2DEG over the whole length of the Hall channel. The magnetic properties of Dy were unintentionally destroyed by air exposure [14] and the modulation was predominantly electrostatic [15] as we shall see below. Modulations with $500 \mathrm{~nm}$ (sample A) and $1000 \mathrm{~nm}$ (sample B) periods, were easily obtained, as shown in figure 1(b). 


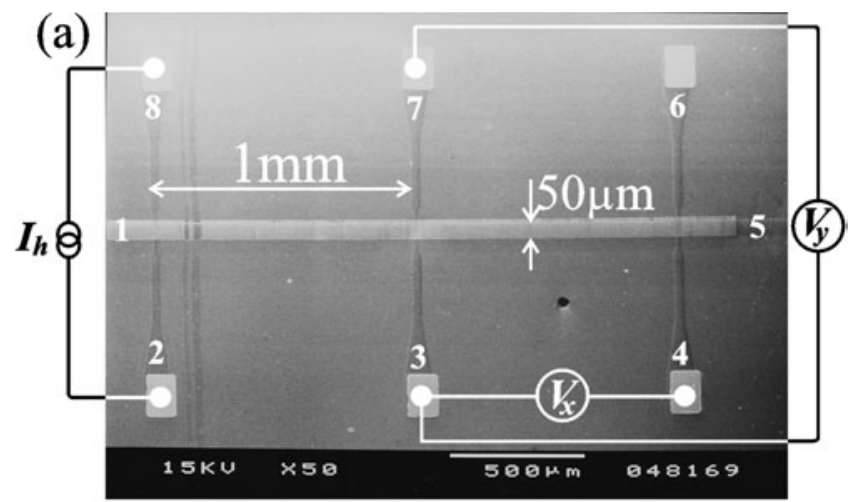

(b)

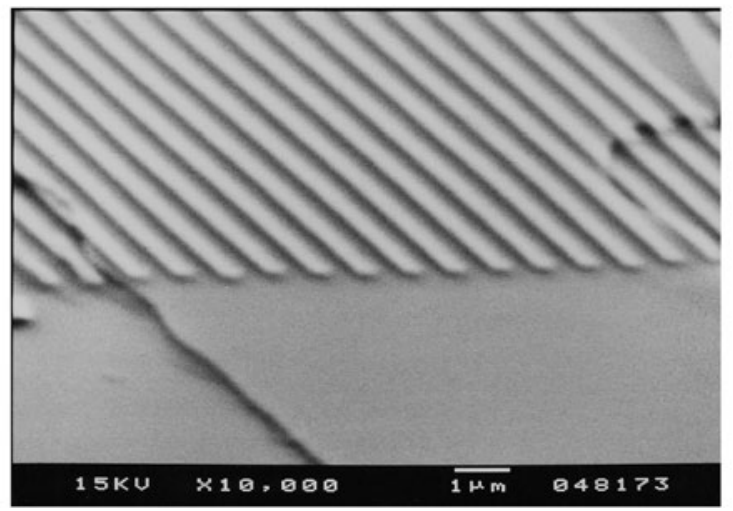

Figure 1. (a) Electron micrograph of a lateral superlattice showing the heating line (2-8) and the voltage probes $(3,4,6,7)$. The probe separation is designed to be larger than the electron energy relaxation length in the 2DEG. (b) A detail of the Dy finger gates that apply a piezoelectric modulation over $4000 \mu \mathrm{m}$ along the Hall bar. Shown here is a $1 \mu \mathrm{m}$-period grating.

The electrical contacts of the Hall bar were used to perform both resistance and thermovoltage measurements. At $4.2 \mathrm{~K}$ the dark electron density and mobility were $n_{\mathrm{s}}=1.3 \times 10^{11} \mathrm{~cm}^{-2}$ and $\mu=320000 \mathrm{~cm}^{2} \mathrm{~V}^{-1} \mathrm{~s}^{-1}$ respectively; these values saturated at $n_{\mathrm{s}}=2.6 \times 10^{11} \mathrm{~cm}^{-2}$ and $\mu=1070000 \mathrm{~cm}^{2} \mathrm{~V}^{-1} \mathrm{~s}^{-1}$ after illumination with an infrared LED. A 1 T superconducting solenoid was used to generate a magnetic field perpendicular to the 2DEG.

In the typical experimental configuration, an ac heating current $(f=7 \mathrm{~Hz})$ was injected through contacts $(2,8)$ in figure 1 (a) and the induced thermovoltage $V_{x}$ was measured across contact pairs $(3,4)$ using lock-in detection at a frequency $2 f$. This method is not a highly efficient way to heat the lattice and the magnitude of the heating current was 1000 times larger than typical current values used in diffusion thermopower experiments [2-4]. We are nevertheless certain that we did measure the phonon drag and not the diffusion thermopower for the three following reasons.

(i) The distance between the voltage probes, $1000 \mu \mathrm{m}$, is larger than the energy relaxation length but smaller than the phonon mean free path (approximately the sample dimensions) [7]. Hot electrons are thermalized long before reaching the first set of voltage probes $1000 \mu \mathrm{m}$ away from the heating line. The energy relaxation length was evaluated as $L=\pi \sqrt{\left(\mu \tau_{\mathrm{ep}} k_{\mathrm{B}} T\right) /(3 e)} \approx 120 \mu \mathrm{m}$ where we used $\tau_{\mathrm{ep}} \approx 10^{-9} \mathrm{~s}$ [16] for the electron-phonon relaxation time. The electron thermal energy at the $(2,8)$ Hall cross 


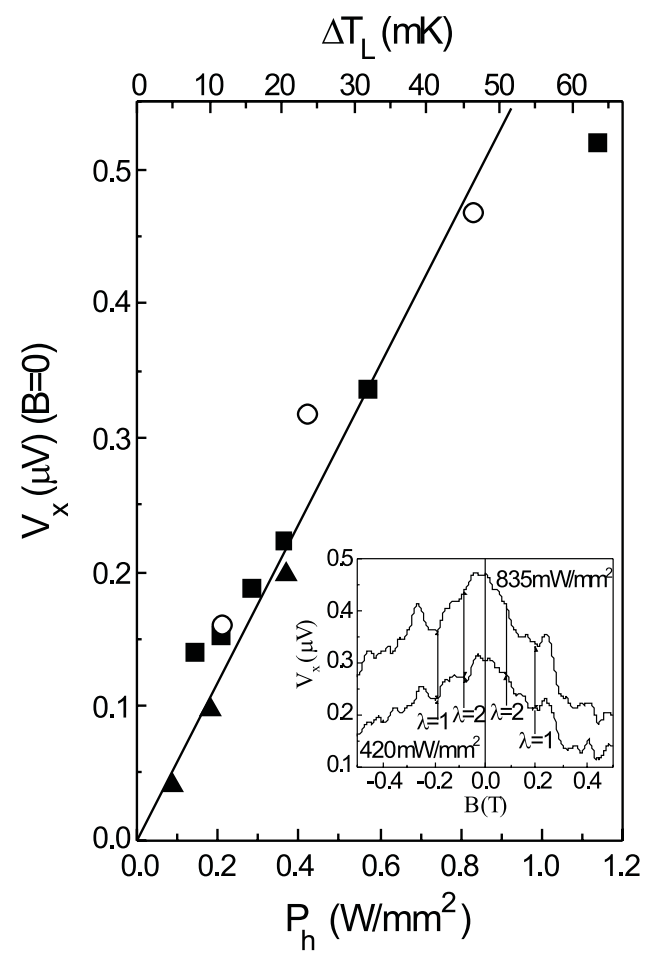

Figure 2. Dependence of the thermovoltage of three separate devices on the Joule power dissipated per unit area of the heating line. Device A: $500 \mathrm{~nm}$ period superlattice (filled triangles) and after scribing the $2 \mathrm{DEG}$ between the heating line $(2,8)$ and voltage probes $(3,7)$ (open circles). Device B: $1000 \mathrm{~nm}$ period superlattice (filled squares). The top $x$-scale shows the increase in lattice temperature at contacts $(3,7)$ relative to the base temperature $(4.2 \mathrm{~K})$. Inset: commensurability thermovoltage oscillations are still seen after device A has been scribed. $n_{\mathrm{s}}=1.4 \times 10^{11} \mathrm{~cm}^{-2}$ (device A) and $n_{\mathrm{s}}=1.9 \times 10^{11} \mathrm{~cm}^{-2}$ (device B).

was calculated as $k_{\mathrm{B}} T=\left(l I_{\mathrm{h}}^{2} \tau_{\mathrm{ei}}\right) /\left(w n_{\mathrm{s}} e \mu\right) \approx 200 \mathrm{meV}$ by taking $l=50 \mu \mathrm{m}$ as the width and $w=10 \mu \mathrm{m}$ as the length of the Hall cross, $I_{\mathrm{h}} \approx 0.1 \mathrm{~mA}$ as the typical heating current and $\tau_{\mathrm{ei}}=m^{*} \mu / e \approx 10 \mathrm{ps}$ as the mobility scattering time.

(ii) We also find good agreement between the magnitude of the measured thermopower and the tabulated values of the PDTP published for similar GaAs heterostructures [7]. Figure 2 plots the thermovoltage $V_{x}$ measured at $B=0$ as a function of the heating power per unit area of the heating line. On the experimental bench, we easily verified that $V_{x}$ increases proportionally to the square of the heating current. In addition, we find the same slope for all three devices studied, once variations in electron density are accounted for in the calculation of the heating power. The lattice temperature at node $(3,7)$ was evaluated indirectly. We first evaluated the rise in lattice temperature in the heating line, node $(2,8)$, by assuming that Joule power sinks in the lattice locally. By equating the electrical power dissipated to the rate at which energy is absorbed in the lattice we obtain the formula: $\Delta T_{\mathrm{L}}=\left(P_{\mathrm{h}} \tau_{\mathrm{ep}}\right) /\left(C_{p} \rho V\right)$ where $C_{p}=0.335 \mathrm{~J} \mathrm{~g}^{-1} \mathrm{~K}^{-1}$ and $\rho=5.36 \mathrm{~g} \mathrm{~cm}^{-3}$ are the heat capacity and crystal density of GaAs respectively. The second step was to evaluate $\Delta T_{\mathrm{L}}$ at node $(3,7), 1000 \mu \mathrm{m}$ away. For this we observed that $V_{x}$ dropped by a factor of 10 when measured across the second nearest pair of probes $2000 \mu \mathrm{m}$ away from the heating line. Assuming an exponential decay of the lattice temperature away from the heater [7] 
allowed us to evaluate $\Delta T_{\mathrm{L}}$ at $(3,7)$ to be 10 times less than $\Delta T_{\mathrm{L}}$ calculated at $(2,8)$. Following this procedure we converted the heating power to a lattice temperature scale shown in the top axis of figure 2. The slope resulting from the linear fit of the data gives a thermopower value $S^{\circ}=110 \pm 40 \mu \mathrm{V} \mathrm{K}^{-1}$ which lies within the range of phonon-drag values, $S^{0}=95-220 \mu \mathrm{V} \mathrm{K}^{-1}$, reported for similar 2DEGs [7, 17]. The tabulated diffusion thermopower in GaAs at $4.2 \mathrm{~K}$ is about ten times smaller than the phonon drag [7]. This is beyond our experimental uncertainty and thus provides an additional argument supporting the observation of phonon drag.

(iii) To eliminate the possibility of electron diffusion we scribed the section of 2DEG between the heater and the voltage probes in device A using a diamond tip. The thermovoltage measured after this operation is shown by the open circles in figure 2 . In the presence of a magnetic field this thermovoltage exhibits the commensurability oscillations shown in the inset to figure 2. Scribing a groove across the 2DEG was a rather crude way of insulating the voltage probes since the groove was also likely to attenuate the transmission of the surface phonon modes. Both the noise and modest amplitude of the $\lambda=1,2$ commensurability oscillations point to this explanation. Nevertheless, by completely eliminating the diffusion contribution, the inset shows that phonon drag is the only possible explanation for the commensurability oscillations.

The electron density was increased incrementally by illuminating the sample with an infrared LED and each time was extracted from the Hall resistance. Above $|B|>0.45 \mathrm{~T}$, a parasitic out of phase voltage component was picked up that restricted our investigation of the thermopower to low magnetic fields. The four-terminal resistance was also measured under the same conditions of temperature and illumination but at a frequency $f=30 \mathrm{~Hz}$ and with a driving current of $100 \mathrm{nA}$.

Figure 3 plots the thermovoltage and the resistance as a function of the magnetic field for sample A. The resistance exhibits the now well known series of $1 / B$ periodic commensurability oscillations [1]. Up to 6 resistance minima are seen at the highest electron density that we label with integer index $\lambda$. It is useful to plot these minima as a function of $1 / B$ in order to clarify the nature of the modulation: magnetic, electrostatic or a combination of both [18]. This result is shown in the inset to figure 3 . The straight lines, each corresponding to one illumination state, were fitted by the relation [1]: $2 R_{\mathrm{c}}=(\lambda-\phi) a$ where $R_{\mathrm{c}}$ is the cyclotron radius at the Fermi level, $a$ is the superlattice period and $\phi$ is the phase of the oscillations and the intercept common to all lines. We find $\phi=+0.18$, a value close to $1 / 4$, the theoretical phase for pure electrostatic oscillations [18]. We therefore conclude that piezoelectric modulation dominates [15]. This is likely to have occurred from the prolonged exposure of dysprosium to air [19].

The thermovoltage, in the top panel of figure 3, exhibits a complicated magnetic field dependence. At the lowest electron density (top curve) the thermovoltage oscillates in phase with the resistance (lower panel, top curve). However, we note a small antisymmetrical component, $V_{x}(B)=-V_{x}(-B)$, that grows dramatically as the electron density increases to $n_{\mathrm{s}}=2.5 \times 10^{15} \mathrm{~m}^{-2}$. At this density, the phase of the thermopower oscillations changes by $180^{\circ}$ through $B=0$. At the same time, the symmetrical component in the thermovoltage is dramatically quenched. For example $V_{x}(B=0)$ drops from $23 \mu \mathrm{V}$ in the dark to nearly zero after full illumination. It is true that part of this drop is accountable to the weaker Joule dissipation in the heating line that reduces the temperature gradient. The lower panel of figure 3 shows $R_{x x}(B=0)$ dropping by $1 / 6$ from 2600 to $430 \Omega$ which is a small variation compared to the near cancellation of $V_{x}(B=0)$. The difference must be ascribed to the decrease in the PDTP with increasing electron density. This behaviour was robust against both thermal cycling between $4 \mathrm{~K}$ and room temperature, and reversal of the temperature gradient. The 


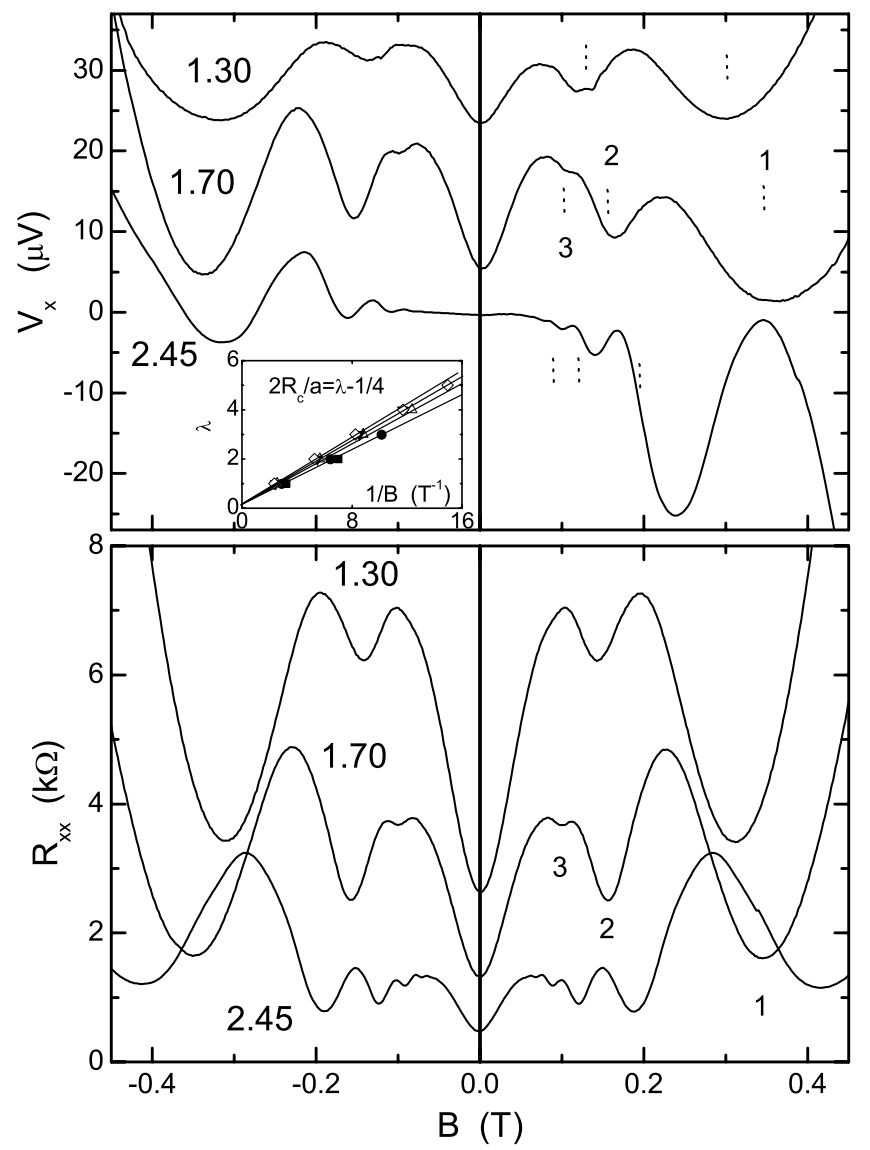

Figure 3. The thermovoltage (top panel) and resistance (lower panel) plotted as a function of the magnetic field for three values of the electron density $\left(n_{\mathrm{s}}=1.3,1.7\right.$ and $\left.2.5 \times 10^{15} \mathrm{~m}^{-2}\right)$ for device A. Inset: the index of the minima in $R_{x x}$ plotted as a function of $1 / B . n_{\mathrm{s}}=1.3$ (filled squares), 1.7 (open triangles) and 2.5 (open diamonds) $\times 10^{15} \mathrm{~m}^{-2}$.

latter was realized by exchanging heater and voltage probes between any of the five contact pairs. We finally recall that the diffusion thermopower is $90^{\circ}$ out of phase with the resistance oscillations $[2,6]$. The fact that our thermovoltage oscillations are either in phase or $180^{\circ}$ out of phase with the resistance concurs with our previous argumentation that we are measuring the phonon drag.

A magnetic field dependence in the phonon drag was unexpected but the antisymmetrical behaviour is puzzling because it cannot be separated in the conventional way as the sum of symmetrical oscillations and a linear magnetic field dependence [20]. The quantities $\left(V_{x}(B)+V_{x}(-B)\right) / 2$ and $\left(V_{x}(B)-V_{x}(-B)\right) / 2$ instead gave two series of oscillations. Sample imperfections such as inhomogeneous electron density or misaligned voltage probes may be ruled out as the resistance oscillations are nearly ideal. One therefore ought to interpret the antisymmetrical oscillations as a property intrinsic to the PDTP in modulated 2DEGs.

\section{Theory and discussion}

An isotropic phonon scattering rate [7, 9, 21] cannot lead to magneto-oscillations in the phonon drag. This is because both the thermoelectric current and the drift current are proportional 
to the conductivity tensor and have the same magnetic field dependence. The requirement that these two currents cancel each other out implies that the temperature gradient and the induced electric field always remain collinear. The assumption that the thermoelectric current is proportional to the conductivity can be understood simply. An electron gains momentum from phonons at a slow rate, $1 / \tau_{\mathrm{ep}} \approx 10^{9} \mathrm{~s}^{-1}$, compared to the rate at which it is randomized by collisions with impurities, $1 / \tau_{\mathrm{ei}} \approx 10^{11} \mathrm{~s}^{-1}$. The acceleration, bending of the trajectory in the magnetic field and momentum scattering are identical to the Drude picture for the conductivity. The thermoelectric current is therefore the conductivity tensor times a constant that accounts for the strength of electron-phonon coupling. A first consequence of this is that there is no transverse induced electric field: $E_{y}(B \neq 0)=0$. Secondly, the longitudinal induced electric field is completely independent of quantum and classical corrections in the conductivity: $E_{x}(B \neq 0)=$ constant [9]. We verified that this model works well with our bare 2DEGs but it starts breaking down as soon as the modulation is applied. The most obvious explanation is that the 1D periodicity introduces a two-fold anisotropy in the electron-phonon scattering rate. The formation of Brillouin minizones means that only a small phonon wavevector, $q=2 \pi / a=1.3 \times 10^{7} \mathrm{~m}^{-1}$, will mediate transitions between opposite edges of the Fermi surface for which transitions the electron-phonon scattering diverges (Kohn anomaly [7]). The wavevector of thermal phonons that one easily calculates to be $q_{\mathrm{th}}=k_{\mathrm{B}} T / \hbar v \approx 10^{8} \mathrm{~m}^{-1}$ at $4 \mathrm{~K}$, spans up to 10 Brillouin minizones. By contrast, the opposite edges of the Fermi surface, in the transverse direction (or in the bare 2DEG), stand separated by $2 k_{\mathrm{F}} \approx 2.3 \times 10^{8} \mathrm{~m}^{-1}$ which is significantly larger that $q_{\mathrm{th}}$. For this reason, it is plausible to assume that Kohn anomalies enhance the scattering probability in the direction of the periodic potential. Anisotropic scattering gives the thermoelectric current a complex magnetic field dependence that is no longer proportional to the conductivity. The drift current has now to compensate the thermoelectric current without the induced electric field being collinear to the temperature gradient. As a result the $E_{x}$ and $E_{y}$ components reflect to some extent the commensurability of the open orbits in the superlattice potential.

The full mathematical derivation of the PDTP has been published elsewhere [22]. Here we concentrate on the physics. We introduce an anisotropic electron-phonon scattering rate in the form of a Fourier expansion in which we retain the lower terms with two-fold angular symmetry:

$$
1 / \tau_{\text {ep }}=1 / \tau_{\text {ep }}^{0}\left\{\frac{a_{0}}{2}+a_{2} \cos (2 \theta)+b_{2} \sin (2 \theta)\right\}
$$

where $a_{0}$ is the isotropic Fourier component, and $a_{2}$ and $b_{2}$ are the anisotropy parameters used to fit the data. $a_{2}$ warps the angular dependence of the scattering rate along the superlattice. If $a_{2}>0$, scattering is high in the direction normal to the stripes otherwise it is high in the direction parallel to the stripes. Having $b_{2} \neq 0$ has the effect of rotating the warping (the principal axes of anisotropy) away from the $x$ - and $y$-axes-we apply the temperature gradient along $x$. We inserted equation (1) into the Boltzmann equation to calculate the charge distribution function and from there the thermoelectric current [22]. The effect of anisotropy is to modify the symmetry of tensor components with respect to the magnetic field. If the temperature gradient is applied along one axis of anisotropy, the diagonal components of the thermopower are symmetric with respect to $B$ whereas the off diagonal components are antisymmetric. If, however, the temperature gradient is tilted with respect to the anisotropy axes, its projection on those axes gives two vector components. These induce a mixing of the symmetric and antisymmetric behaviour in each thermopower tensor component. Note that this mixing is more complicated than a linear superposition due to cross product terms between the vector components of the temperature gradient [22]. In the simpler situation 


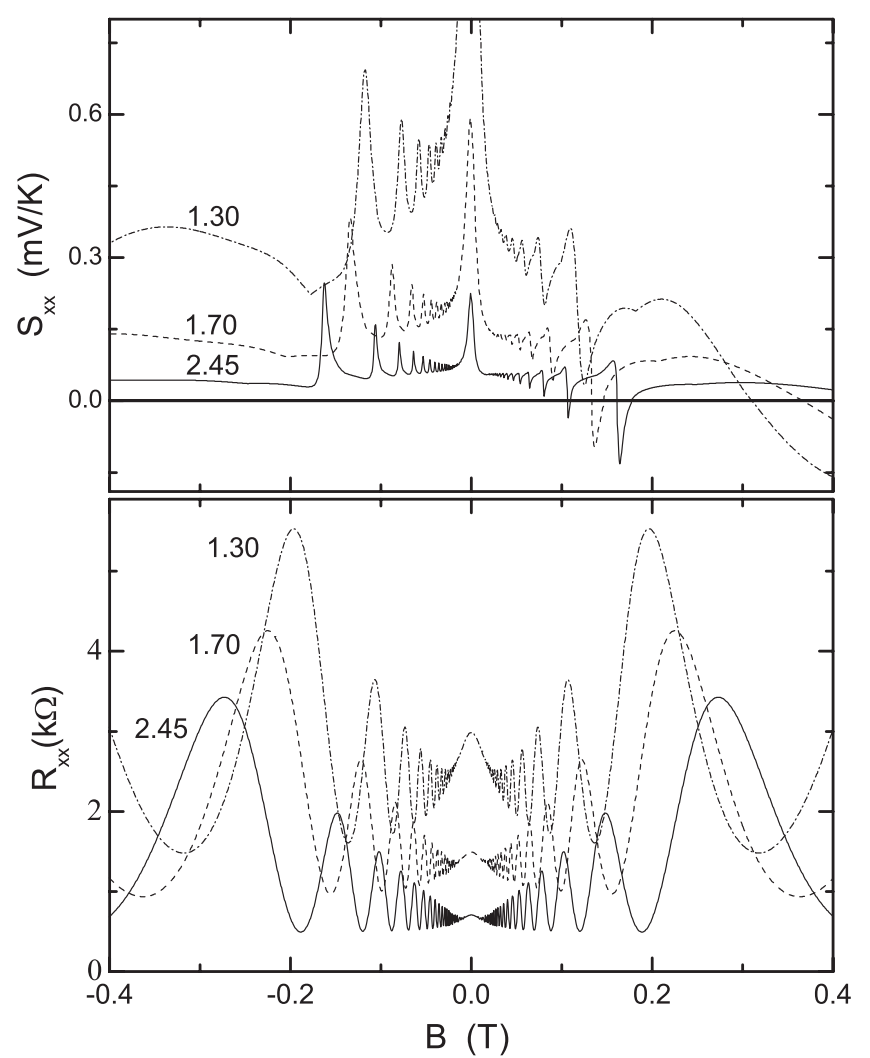

Figure 4. The PDTP oscillations solution of the Boltzmann equation. The electron-phonon scattering rate is anisotropic in the plane of the 2DEG with Fourier components $a_{0}=a_{2}=+45$ and $b_{2}=+11$ (see text). $b_{2} \neq 0$ indicates a finite angle between the temperature gradient and one of the principal axes of anisotropy that is responsible for the asymmetric part of $S_{x x} . a_{2} \neq 0$ is responsible for the symmetric commensurability oscillations and more generally the magnetic field dependence of $S_{x x}$. These are the only fitting parameters of the model. A tiny tilt angle (dashed/dot dashed curves) is sufficient to induce asymmetric oscillations of the right magnitude. Here $a=500 \mathrm{~nm}, \tau=14,21$ and $38 \mathrm{ps}$ (for $n_{\mathrm{s}}=1.3,1.7$ and $2.5 \times 10^{15} \mathrm{~m}^{-2}$ respectively), $a_{0}=a_{2}=45, b_{2}=11$ and $S^{0}=110 \mu \mathrm{V} \mathrm{K}^{-1}$.

where the anisotropy axes are aligned with the axes of the coordinates $\left(b_{2}=0\right)$, the off diagonal component of the thermopower vanishes whatever the magnetic field. In this case, the longitudinal oscillations are symmetric and their amplitude is proportional to $a_{2}$.

The Boltzmann equation was solved analytically [22] and the parameters $a_{2}$ and $b_{2}$ obtained by fitting the numerical results to the experimental plots of figure 3 . All other parameters of the calculation are either measured from the bare 2DEG or given by the structural parameters. The best fit is obtained for $a_{2}=45 \pm 20$ and $b_{2}=11 \pm 5$, see figure 4 . The positive value of $a_{2}$ demonstrates that acoustic phonon scattering is enhanced by the periodic potential which supports our argument on Kohn anomalies. The increase in $n_{\mathrm{s}}$ from 1.3 to $2.5 \times 10^{11} \mathrm{~cm}^{-2}$ reduces the theoretical phonon drag by a factor of 5 in figure 4 which is close to the experimental dependence of the thermopower in figure 3. This drop is because electrons become less affected by anisotropic scattering as their kinetic energy increases. It is interesting to note that increasing the impurity scattering time $\tau$ has no effect here. On the one hand, it enhances the cyclotron lifetime and thus further perturbs the guiding centre 


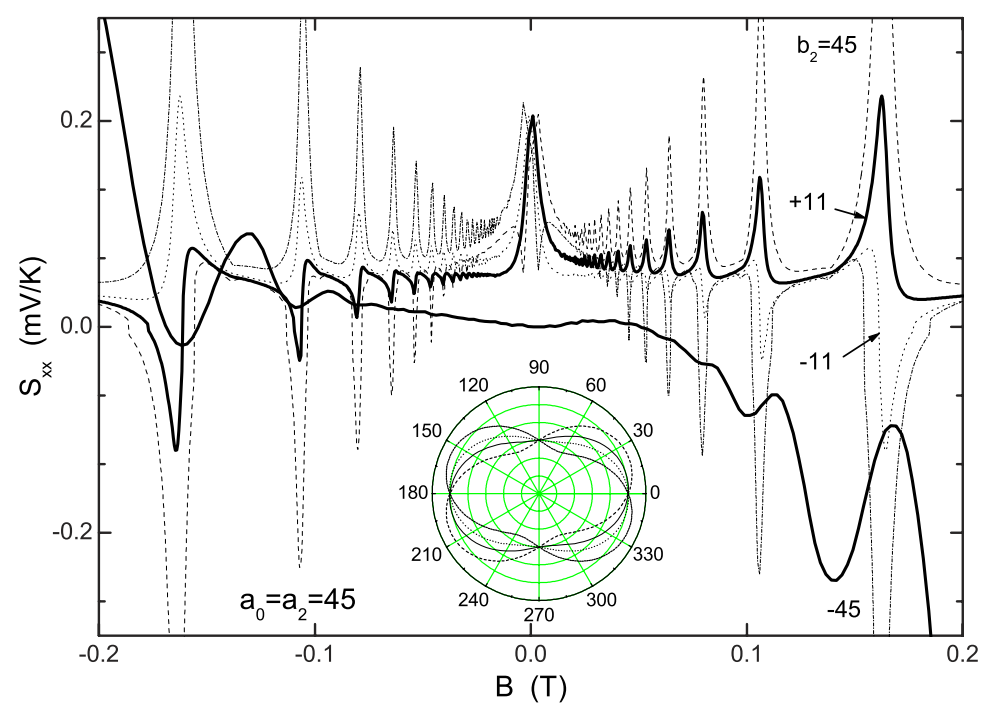

Figure 5. The influence of misalignment between the temperature gradient and a principal axis of anisotropy (parameter $b_{2}$ ) on the thermopower oscillations: $b_{2}=+45$ (dashed curves) +11 (thick solid curve), -11 (dotted curve) and -45 (short dashed curve). The effect of $b_{2}$ on the electron-phonon scattering rate is shown in the inset where the $0^{\circ}-180^{\circ}$ line is the superlattice direction.

(This figure is in colour only in the electronic version)

drift; on the other hand, the longer mean free path averages anisotropy over the length of the electron trajectory ${ }^{4}$. Our anisotropic theory is superior to the isotropic result presented in the introduction since it explains the dependence of the thermopower on $n_{\mathrm{s}}$. The anisotropic theory also explains the development of antisymmetrical oscillations when $n_{\mathrm{s}}$ increases, including the change in sign of the PDTP at $n_{\mathrm{s}}=2.5 \times 10^{11} \mathrm{~cm}^{-2}$. Figure 5 shows the effect of $b_{2}$ on the antisymmetrical behaviour of the thermopower while the inset plots the corresponding contours of the electron-phonon scattering rate calculated according to equation (1). The $0^{\circ}-180^{\circ}$ and $90^{\circ}-270^{\circ}$ axes are the $x$ - and $y$-axes respectively. The optimum fit $b_{2}=+11 \pm 5$ suggests that the temperature gradient is misaligned by $6^{\circ}$ with respect to the superlattice axis. This is an acceptable assumption considering that the distribution of ballistic phonons reaching node $(3,7)$ in figure 1 has an angular aperture of $(5 / 100) \times 180 / \pi=3^{\circ}$. Our theory presents the advantage (and limitations) of being almost entirely analytical. The formation of skipping orbits that are responsible for the low field magnetoresistance and magneto-thermopower in figure 3 cannot accounted be for by the present model [23]. This is the reason why the calculated curves show a maximum rather than a minimum at $B=0$ in figure 4 . A more accurate description of the experiment could be obtained by including in the model the real piezoelectric potential which is usually more complex than a sine function [15]. Secondly, a more accurate description of the anisotropy would require computing the effect of higher angular harmonics with two-fold symmetry, not just the $a_{2}$ and $b_{2}$ components. Finally, although the relative values of $a_{2}$ and $b_{2}$ are satisfactory, their absolute values are too large by a factor of 20. This discrepancy is likely to arise from our indirect estimate of $\Delta T_{\mathrm{L}}$.

4 Mathematically speaking the $\alpha_{m}$ coefficients in equation (15) of [22] vanish at higher electron density. 


\section{Summary}

In summary, we have measured commensurability oscillations in the PDTP of a lateral superlattice. We fitted our data with our recent theory [22] that builds upon the isotropic Boltzmann theory to predict the effect of an anisotropic electron-phonon scattering induced by Brillouin zone folding on the thermoelectric properties of lateral superlattices. The longitudinal temperature gradient induces symmetrical thermopower oscillations that are in phase with the resistance oscillations. A slight misalignment of the temperature gradient with a principal axis of anisotropy induces an effective transverse temperature gradient that gives commensurability oscillations antisymmetrical in $B$. The theory for this effect is in full agreement with the dependence on electron concentration. It may also explain the phase of the commensurability oscillations at high temperature [24]. It is hoped that the present demonstration of magnetic field dependent effects in the PDTP together with the recent observation of the universal quantum of thermal conductance [25-27] will stimulate further investigation of the PDTP of mesoscopic systems.

\section{Acknowledgments}

We thank Professor S Bending for granting experimental facilities and acknowledge the support of INTAS99-1661.

\section{References}

[1] Weiss D, von Klitzing K, Ploog K and Weimann G 1989 Magnetoresistance oscillations in a two-dimensional electron gas induced by a submicrometer periodic potential Europhys. Lett. 82

[2] Kearney M J, Syme R T and Pepper M 1991 Observation of quantum interference in thermoelectric transport Phys. Rev. Lett. 661622

[3] Godijn S F, Moller S, Buhmann H, Molenkamp L W and van Langen S A 1999 Thermopower of a chaotic quantum dot Phys. Rev. Lett. 8214

[4] Pogosov A G, Budantsev M V, Uzur D, Nogaret A, Plotnikov A E, Bakarov A K and Toropov A I 2002 Thermopower of a multiprobe ballistic conductor Phys. Rev. B 66201303

[5] Taboryski R, Brosh B, Simmons M Y, Ritchie D A, Ford C J B and Pepper M 1995 Magnetothermopower oscillations in a lateral superlattice Phys. Rev. B $\mathbf{5 1} 17243$

[6] Peeters F M and Vasilopoulos P 1990 Thermomagnetic transport coefficients of a periodically modulated twodimensional electron gas Phys. Rev. B 429

[7] Gallagher B L and Butcher P N 1992 Handbook on Semiconductors (Amsterdam: Elsevier) chapter 14, pp 721-816

[8] Fletcher R 1999 Magnetothermoelectric effects in semiconductor systems Semicond. Sci. Technol. 141

[9] Miele A, Fletcher R, Zaremba E, Feng Y, Foxon C T and Harris J J 1998 Phonon-drag thermopower and weak localisation Phys. Rev. B 5819

[10] Tieke B, Fletcher R, Zeitler U, Henini M and Maan J C 1998 Thermopower measurements of the coupling of phonons to electrons and composite fermions Phys. Rev. B $\mathbf{5 8} 4$

[11] Beenakker C W J 1989 Guiding-center-drift resonance in a periodically modulated two-dimensional electron gas Phys. Rev. Lett. 6217

[12] Winkler R W, Kotthaus J P and Ploog K 1989 Landau-band conductivity in a two-dimensional electron system modulated by an artificial one-dimensional superlattice potential Phys. Rev. Lett. 6210

[13] Gerhardts R R, Weiss D and von Klitzing K 1989 Novel magnetoresistance oscillations in a periodically modulated two-dimensional electron gas Phys. Rev. Lett. 6210

[14] Spence D J, Noakes T C Q, Bailey P and Tear S P 2001 Investigation of a two-dimensional dysprosium germanide on ge(111): a medium-energy ion-scattering study including direct observation of reversal of top-layer buckling upon adsorption of hydrogen Phys. Rev. B 63049901

[15] Larkin I A, Davies J H, Long A R and Cusco R 1997 Theory of potential modulation in lateral surface superlattices. ii. piezo-electric effect Phys. Rev. B 5615242

[16] Kent A J 1988 Hot Electrons in Semiconductors (Oxford: Clarendon) chapter 4, pp 81-107 
[17] Fletcher R, Harris J J, Foxon C T, Tsaousidou M and Butcher P N 1994 Thermoelectric properties of a verylow-mobility two-dimensional electron gas Phys. Rev. B $\mathbf{5 0} 20$

[18] Peeters F M and Vasilopoulos P 1993 Quantum transport of a two-dimensional electron gas in a spatially modulated magnetic field Phys. Rev. B 471466

[19] Spence D J, Noakes T C Q, Bailey P and Tear S P 2001 Investigation of a two-dimensional dysprosium germanide on ge(111): a medium energy ion-scattering study of top layer buckling upon adsorption of hydrogen Phys. Rev. B 63049901

[20] Pogosov A, Budantsev M V, Kibis O V, Pouydebasque A, Maude D K and Portal J C 2000 Thermomagnetic effect in a two-dimensional electron system with an asymmetric quantising potential Phys. Rev. B 6123

[21] Herring C 1954 Theory of the thermoelectric power of semiconductors Phys. Rev. 951163

[22] Nogaret A 2002 Signature of anisotropic phonon-drag in the thermopower of periodically modulated structures Phys. Rev. B 66125302

[23] Beton P H, Alves E S, Main P C, Eaves L, Dellow M, Henini M, Hughes O H, Beaumont S P and Wilkinson C D W 1990 Magnetoresistance of a two-dimensional electron gas in a strong periodic potential Phys. Rev. B 4214

[24] Mitzkus C, Kangler W, Weiss D, Wegscheider W and Umansky V 2002 Anomalous temperature dependence of commensurability oscillations in one- and two-dimensional lateral superlattices Physica E 12208

[25] Schwab K, Henriksen E A, Worlock J M and Roukes M L 2000 Measurement of the quantum of thermal conductance Nature 404974

[26] Sales B C 2002 Smaller is cooler Science 2951248

[27] Feng Q, Yang P and Guo H 2002 Four-terminal thermal conductance of mesoscopic dielectric systems Phys. Rev. Lett. 89175901 